\title{
Hierarchical Clustering based Energy Efficient Protocols for Wireless Sensor Networks: A Review
}

\author{
Ritika \\ M.Tech(CSE) Scholar \\ Department of Computer Science \\ \& Engineering \\ DAVIET, Jalandhar
}

\author{
Harpreet Kaur \\ Associate Professor, Head \\ Department of Computer Science \\ \& Engineering \\ DAVIET, Jalandhar
}

\author{
Gursewak Singh \\ Assistant Professor \\ Department of Computer Science \\ \& Engineering \\ DAVIET, Jalandhar
}

\begin{abstract}
Wireless sensor network are geographically distributed selfdirected to scrutinize environmental and physical conditions for instance like noise, pressure, temperature and so on .Energy saving to protract the network life is one of the main design issue during developing a new Clustering Based Energy Efficient Protocol for wireless sensor networks. Clustering is the mechanism for multi hop WSN to acquiring scalability, sinking energy utilization and amplify the life time of a network to attain improved network performance. In this paper focus is mainly driven on the survey of the Hierarchical Clustering Based Energy Efficient Protocols for WSN.
\end{abstract}

\section{Keywords}

Clustering, WSNs, Energy efficiency, Network Lifetime.

\section{INTRODUCTION}

Wireless Sensor Networks - A network of devices that can communicate the information accumulated from a supervised field through wireless links. The WSN is an intelligent and inexpensive solution that enables the efficiency and reliability enhancement of many industrial applications such as security surveillance, home and building automation, and smart grids. In this network, nodes are connected with wireless mediums such as air/atmosphere to transmit the data between different nodes. Commonly transfer mediums used are microwave communication, radio wave communication, satellite communication and so on.[1]

A WSN consists of spatially distributed sensors known as Sensor Nodes that sense and monitor the environmental surroundings along with communication between other nodes. Each of these scattered sensor nodes have the potential to examine specific conditions of atmosphere and evaluate those conditions with collected data linked to phenomenon where sensors are tiny devices with restricted resources such as restricted battery power, minute computing potential, tremendously low data rates, low memory, irregular link quality, low bandwidth processing. Large numbers of sensors are deployed in harsher environment, it is important that the distributed computation should be robust and fault-tolerant.[3]

One of the main concerns during development of the WSN is to lengthen their lifetime. In many applications, a sensor node is power-driven by a finite energy sources like a battery or a super capacitor that curbs the WSNs' lifetime. The renewable energy sources such as solar or wind have been investigated and integrated with the sensor nodes recently for longer operation. However, the intermittent nature of these sources still has a significant consumption of the WSNs need to be taken into account when planning the network operation.[4]
In WSNs each sensing node has a Radio Transceiver for sending the information (Transmitter) and receiving the Information (Receiver), an Antenna for providing connectivity to the Network, a Micro Controller for controller for controlling the operation of Sensor Node, an Electronic Circuit for interfacing with other Sensors and an Energy Source usually a Battery as each element utilizes some form of energy to carry out its operation.

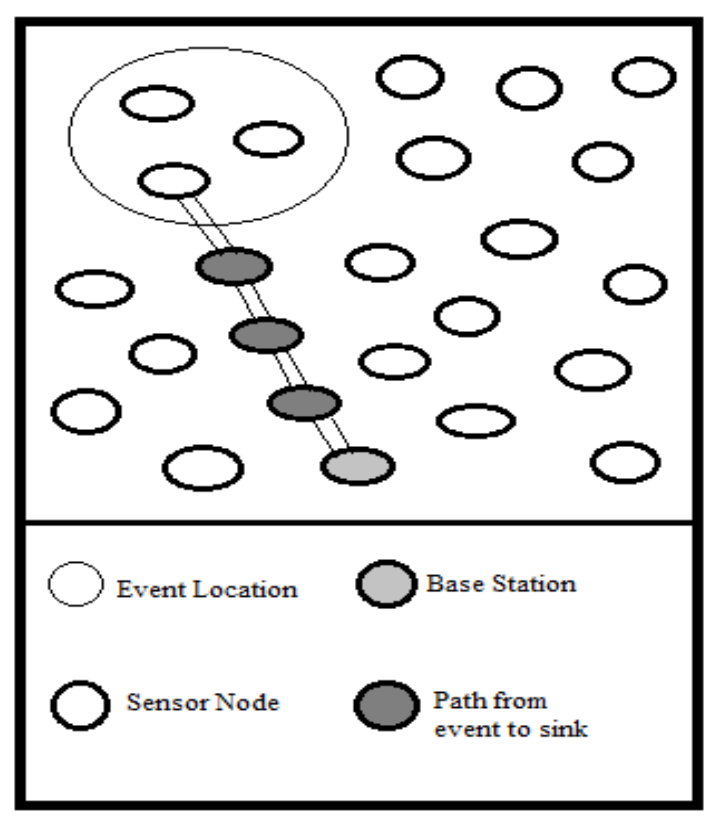

Figure1. Wireless Sensor Network Environment

Sensor Nodes can be considered as small computers in the terms of their interfaces and components. They generally have a processing unit with limited computational capabilities and limited Memory, Sensors, a Transceiver and a Power Source usually a Battery.[2] These nodes can share the data either through Routing or through Flooding. The components of network (fig 1.) as follows:

- Sensor Node: A Sensor Node can take on multiple roles in a network, such as simple sensing; data storage; routing; and data processing.

- Clusters: Clusters are the organizational unit for WSNs. The dense nature of these networks requires the need for them to be broken down into clusters to simplify tasks such a communication.

- Base Station: The base station is at the upper level of the hierarchical WSN. It provides the 
communication link between sensor network and end-users.

- End Users: The data in a sensor network can be used for a wide-range of application. [5]

The major characteristics of Wireless Sensor Networks are:

a) Power Consumption Constraint.

b) Reliability/Resilience to cope with Node Failures.

c) Mobility of nodes.

d) Heterogeneity of Nodes.

e) Scalability.

f) Cross layer design to improve Quality of Service.

g) Ability to withstand harsh environmental conditions.

\section{CLUSTERING}

Wireless sensor networks are facing issue these days is how to hoard the energy consumption to lengthen the network longevity. Quite a few WSN applications require only the combined value to be submitted to the viewer. Sensor nodes aggregate all the data from different sources and send to the viewers. In order to support data aggregation through well-organized network group, nodes can be divided into a number of small groups called Clusters.[7]

Every cluster has a controller, which is as a Cluster Head $(\mathrm{CH})$, and has numerous component nodes. The cluster formation process ultimately leads to a two-level where the $\mathrm{CH}$ nodes form the advanced level and the cluster-member nodes form the minor level. The sensor nodes from time to time transmit their data to the equivalent $\mathrm{CH}$ nodes. The $\mathrm{CH}$ nodes combine the data and transmit it to the base station (BS) either directly or during the intermediate communication with other $\mathrm{CH}$ nodes.

A general resolution in order to stabilize the energy utilization among all the network nodes is time to time re-elect new $\mathrm{CHs}$ in each cluster. The BS is the data processing point for the data received from the sensor nodes, and where the data is accessed by the end user. It is generally well thought-out predetermined and at a far distance from the sensor node. The $\mathrm{CH}$ nodes essentially act as gateways between the sensor nodes and the BS.[8]

A primary metric for evaluating the performance of a sensor network as follows:

- Load balancing.

- Fault-tolerance

- Increased connectivity and Dwindled delay.

- Maximum network lifetime.

- Minimum energy consumption.

- Highly robust.

- Quality of service.

\section{ENERGY EFFICIENCY IN WIRELESS SENSOR NETWORK}

Sensor network contains a huge number of tiny, low-cost apparatus with sensing, transmitting and processing potentials. The major aim of the procedure is to examine a region as well as gather plus relay details to the sink node or else the set of the sink nodes, known as BS- Base Station. Promoting the information to the Base Station is probable in two manners: by the use of direct and multi hop communication. First case- Every sensor broadcast its data straight to sink. Second case- The sensors are conversing with neighbors, which forward the data on the route of sink. Sensor networks are also categorized through the periodicity of information transmission. Each node transmits message in the time-driven network, whereas a node transmits message in an even-driven network only while sensing the phenomenon.[6] The $3^{\text {rd }}$ class is query-driven access, where the sensors transfer data only once receiving a query as of the Base Station. There exist hybrid networks also that merge the earlier three models.

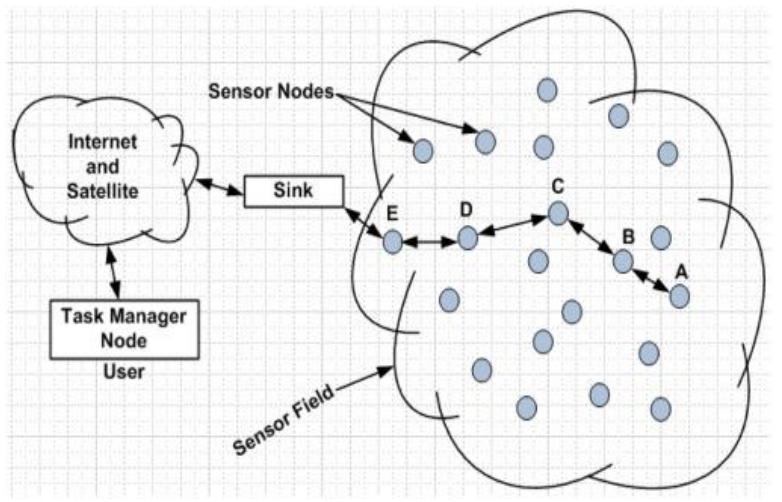

Figure2. Clustering in sensor nodes.

\section{TAXONOMY OF CLUSTERING APPROACHES}

\subsection{Leach (Low energy adaptive clustering hierarchy):}

LEACH is the very frequent energy efficient hierarchical clustering algorithm for WSNs that was developed for dipping power consumption. LEACH is a protocol in which most nodes transmit the data to cluster heads, and the cluster heads combine and compresses that data and forwards it to the base station. LEACH divides the whole network into numerous clusters, which are designed by using leach coordination and control. This will not only reduce the amount of data to be transmitted to the $\operatorname{sink}(\mathrm{BS})$, but also to make routing and data distribution more flexible and robust.. Leach selects arbitrarily the nodes $\mathrm{CH}$ cluster heads also allocate function to dissimilar nodes by utilizing round robin strategy to make sure fair energy indulgence among nodes. To minimize the amount of the data sent to the base station, Ch-cluster head collective the data composed of member node linked with their own cluster also on the collective packet to a base station. This algorithm's working involves two phases:

a. $1^{\text {st }}$ phase : In this phase, clusters are arranged in the form of network structure. This phase is known as setup phase. In set-up phase every node of the network takes decision regarding to be the cluster head or not be the cluster head. Formation of the Cluster head is based on the planned percentage of the Cluster Head $(\mathrm{CH})$ to a network end cluster node have turn into the cluster head meant for the number of times. A choice to become the cluster head is prepared by node choosing the arbitrary number among 0 and 1 . Sensor node would convert into the cluster head if number was greater than the following threshold (1): 


$$
\begin{array}{ll}
T(n)=\frac{P}{1-P \times\left(r \bmod \frac{1}{P}\right)} & \forall n \in G \\
T(n)=0 & \forall n \notin C
\end{array}
$$

Here $\mathrm{P}$ is a preferred percentage of the $\mathrm{CHs}$, is a current round, and also is a set of nodes which are not elected as a $\mathrm{CHs}$ in last $1 / \mathrm{p}$ round.

$$
2^{\text {nd }} \text { phase: }
$$

This phase is called the steady state phase. Here data is send to Base Station also compressing the sensing of the data is completed.

\subsection{HEED (Hybrid Energy-Efficient Distributed clustering):}

HEED protocol is a wireless multi-hop clustering protocol that causes the energy-efficient clustering routing by straight reflection of power. Heed is dissimilar from LEACH in the process of selection of cluster head, cluster heads were not chosen arbitrarily The Cluster Heads are chosen by two factors in HEED, as residual energy as well as intra-cluster transmission price of nodes. We suppose Cprob is the most favorable percentage which can't be computed earlier in LEACH protocol. Probability that the node will turns into $\mathrm{CH}$ is :

$$
C H_{\text {prob }}=C_{\text {prob }} \times \frac{E_{\text {resiaual }}}{E_{\max }}
$$

Here Eresidual is an predictable current energy of a node also Emax is the reference highest energy that is usually equal for every node in a network.

The magnitude of $\mathrm{CH}$ prob , though is not permissible to fall lower than a certain threshold which is chosen as inversely proportional to the Emax.

\subsection{TL-LEACH (Two-Level Hierarchy LEACH):}

In TL-Leach protocol, the cluster head collect the information from nodes same as LEACH protocol, however it doesn't transmit data to Base Station directly, instead it utilizes a portion of the Cluster Head which is placed among the BS and the $\mathrm{CH}$ also called as Relay Station. Cluster Heads at the top known is primary $\mathrm{Ch} i$, second $\mathrm{CHs}$ offered from secondary $\mathrm{CHi}$, also ordinary nodes. An algorithm maybe cleared from necessary steps as follows:

- First step is known as Advertisement phase, where every node make decision regarding it will turn into primary cluster head or not, secondary $\mathrm{CHs}$ or ordinary node in each round similar to LEACH protocol. IF a node chooses to turn into primary $\mathrm{CH}$ in that case node advertise additional nodes by utilizing CSMA-carrier sense multiple accesses.

- Second step that is known as Cluster set-up phase, every secondary $\mathrm{CH}$ build decision so as to which primary $\mathrm{CH}$ this node needs to fit in also transmit signal like an advertisement memo towards its primary $\mathrm{CH}$. According to the above method each ordinary node must choose that which secondary $\mathrm{CH}$ this node need to belong also send these detail by using differing message.

- Third step is known as Schedule creation, each primary $\mathrm{CH}$ generates a TDMA time table by allocating each node in their segments as the time period to send out. Every primary selects the CDMA code also transmit message to every node at second stage in its set to consume code and with using above technique each secondary $\mathrm{CH}$ can transmit its data to the ordinary node into its cluster by both the schedule and the code from primary $\mathrm{CH}$.

- Forth step is called as Data transmission phase. In this, clusters are created and each node can transmit by using TDMA time table planned through its primary $\mathrm{CH}$.

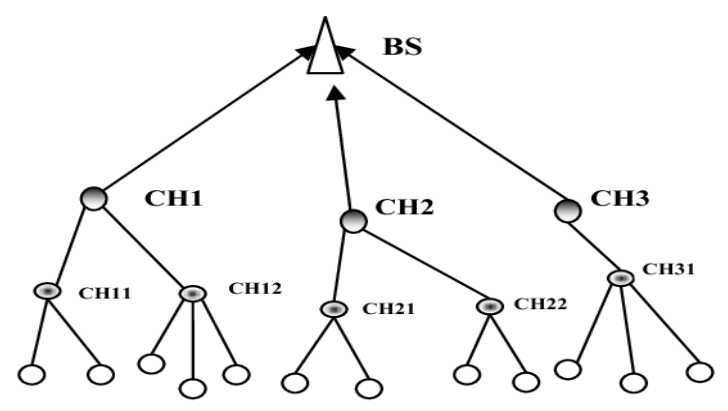

Figure 3 TL-LEACH

\subsection{TEEN(Threshold Sensetive Energy \\ Efficient Sensor Network Protocol):}

TEEN is the hierarchical protocol and its main principle is to contract unexpected alteration in sensed features like energy. The protocol combines a tree based method in the line by the data-centric method. The nodes adopt their environnment firmly, but the power consumption in the TEEN could probably be greatly smaller then that as in a proactive network, because data transmission among nodes is completed in less regular way.[9] TEEN executes the operation by giving to threshold roles:

- Hard Threshold: It is the threshold function magnitude for sensed part. It is an absolute value by which a node which had sensed this magnitude must transmit towards its transmitter also informs to its $\mathrm{CH}$.

- Soft Threshold: It is a slightly different in a value of a sense attribute that makes a node to transmit towards its transmitter.

\subsection{APTEEN (The Adaptive Threshold Sensetive Energy Efficient Sensor Network Protocol ):}

The main aim of APTEEN protocol is to send out both periodic data also reacting to time significant events. APTEEN the hybrid protocol which changes the threshold values or periodicity utilizes in TEEN as essential according to clients and the sort of an application necessary. It is a cleared query based system which allows three kind of queries: One-time, Persistent and Historical. Sink node transmit the following parameters in the APTEEN protocol:

- Attribute: It a group of the physical variables which the client is focused in attaining data.

- Threshold Function: It is the combination of both soft and hard threshold. Soft Threshold was a slight transformation in a value which activates a node to 
transmit data again. While, Hard Threshold was a value which is used to activate data to a sink node.

- Schedule: The Time-Division Multiplexing plan is utilized for allocating a slot to all nodes.

- Count Time: Count Time is the utmost time limit among two common outputs transmits via node. It could be the multiple of TDMA schedule duration and it could as well accounts for proactive attribute.[10]

\subsection{EEHC (Energy Efficient Hierarchial cluster):}

EECH protocol each sensor node is a network turn into cluster head by probability $p$ also sends data to other nodes so as this node is a cluster head inside the range of the radio and these $\mathrm{CH}$ cluster heads is called volunteer cluster heads $(\mathrm{CH})$. Any sensor node which gets such data as well as not a $\mathrm{CH}$-cluster-head in a given range chooses a cluster of a closet cluster-head. A few sensor nodes which was not a $\mathrm{CH}-\mathrm{Cluster}$ head or else has to selected any cluster would turns into a $\mathrm{CH}$-cluster head. These kind of clusters are called a forced cluster heads. Since we have delimited information transmitting to the $k$ hops, and if the sensor nodes was not able to find $\mathrm{CH}$ transfering data with in time period $t$, it can wrap up that it was not in the range of the $k$-hops of further volunteer cluster heads also will appears to be the forced cluster heads. Restricting the amount of hops thus allows a cluster head to manage their communication.

\section{CONCLUSION}

Wireless Sensor Networks have been developed as well as applied to commercial, industrial, defense and civil sector applications. We have discussed and overview of exiting energy efficiency protocols and techniques in clustering environment. Finally it is concluded from the surveys that most of in this work we modeled the act of protocols for cluster head selection by LEACH, HEED and other hierarchical protocols in static network of network areas. In this, we consider different parameters such as cluster formation, selection of head, regional energy source for isolated nodes battery lifetime, noise and location uncertainty on wireless link reliability all these techniques better in good predesigned environment conditions so as sink relocation has been done by which energy consumption problem has been solved.

\section{REFERENCES}

[1] Juneja, D., Sharma, A., Kumar, A.: A Novel Application Of Extended Kalman Filter For Efficient Information Processing In Subsurfaces. International Journal of Computer Applications 17(2), 28-32 (2011); Published By FCS (Foundation of Computer Science, USA). ISSN: 09758887.

[2] I.F. Akyildiz, W. Su, Y. Sankarasubramaniam, E. Cayirci, "Wireless Sensor Networks: A Survey", Computer Networks, vol. 38, no. 4, pp. 399-422, 2002.

[3] Al-Sakib, Pathan, K., Lee, W., Hyung, Hong, S., Choong.: Security in Wireless Sensor Networks: Issues and Challenges. In: ICACT 2006.

[4] J. Yick, B. Mukherjee, D. Ghosal, "Wireless Sensor Network Survey", Computer Networks, vol. 52, no. 12, pp. 2292-2330, 2008.

[5] R.Mitra, D. Nandy, "A Survey on Clustering Techniques for Wireless Sensor Network", Int. J. of Research in Computer Science Vol. 2 Issue 4 (2012).

[6] R.Mitra, D. Nandy, "A Survey on Clustering Techniques for Wireless Sensor Network", Int. J. of Research in Computer Science Vol. 2.

[7] M.Guerroumi,N.Badache,S.Moussaoui,"Mobile sink and power management for efficient data dissemination in wireless sensor networks", Springer Science+Business Media New York 2014

[8] Chu-Fu Wang, jau-Der Shih, Bo-Han Pan and Tin-Yu Wu, "A Network Lifetime Enhancement method for Sink Relocation and Its Analysis in Wireless sensor Networks", IEEE Senosrs Journal, (2014), Vol. 14, No. 6, 1932 - 1943.

[9] YI Gao \& Wei Dong "COPE: Improving Energy Efficiency with Coded Preambles in Low Power Sensor Networks" 2015, IEEE Transaction and Industrial Informatics.

[10] Sheetal Watkar, "Review Paper on Energy Efficient Protocol in Wireless Sensor Network "Int. Journal of Engineering Research and Applications, Vol. 5, Issue 3, ( Part - 3) March 2015,pp.107-11 